\title{
Acupuntura auricular na redução da dor em idosos: revisão integrativa
}

\author{
Ear acupuncture for pain reduction in the elderly: integrative review \\ Acupuntura de oído para la reducción del dolor en los ancianos: revisión integradora
}

\section{Resumo}

Objetivo: Analisar as evidências disponíveis na literatura sobre o uso da acupuntura auricular na redução da dor em idosos. Metodologia: Revisão integrativa da literatura realizada nas bases MEDLINE, LILACS, Web of Science, Scopus, ScienceDirect e CINAHL, por meio dos descritores "idoso" (aged), "acupuntura auricular" (auricular acupuncture OR ear acupuncture) e "redução da dor" (pain). A busca inicial encontrou 179 estudos, foram incluídos artigos originais, disponíveis na íntegra, em qualquer idioma e não foi adotado recorte temporal. Após a revisão de títulos e resumos, 15 permaneceram para análise do texto completo. Por fim, 11 estudos foram incluídos na revisão. Resultados: A eficácia da acupuntura auricular no controle e tratamento da dor foi evidenciada em populações diversas, tais como idosos oncológicos, indivíduos submetidos a procedimentos cirúrgicos e com algum tipo de dor. Conclusão: Apesar do número limitado de estudos com idosos, das populações diversificadas e dos diferentes protocolos de intervenção, recomenda-se que a AA associada ou não a outros tratamentos pode contribuir para a redução da dor em idosos.

Palavras-chave: Idoso; Acupuntura auricular; Redução da dor.

\begin{abstract}
Objective: To analyze the evidence available in the literature on the use of auricular acupuncture to reduce pain in the elderly. Methodology: Integrative literature review performed in MEDLINE, LILACS, Web of Science, Scopus, ScienceDirect and CINAHL databases, using the descriptors "elderly" (aged), "auricular acupuncture" (auricular acupuncture OR ear acupuncture) and "pain reduction" (pain). The initial search found 179 studies, original articles were included, available in full, in any language, and no time frame was adopted. After reviewing titles and abstracts, 15 remained for full text analysis. Finally, 11 studies were included in the review. Results: The effectiveness of auricular acupuncture in pain control and treatment was evidenced in different populations, such as oncologic elderly, individuals undergoing surgical procedures and with some type of pain. Conclusion: Despite the limited number of studies with the elderly, the diverse populations and the different intervention protocols, it is recommended that AA associated or not with other treatments can contribute to pain reduction in the elderly.
\end{abstract}

Keywords: Elderly; Ear acupuncture; Pain reduction.

\section{Resumen}

Objetivo: Analizar la evidencia disponible en la literatura sobre el uso de la acupuntura auricular para reducir el dolor en el anciano. Metodología: Revisión integrativa de la literatura realizada en las bases de datos MEDLINE, LILACS, Web of Science, Scopus, ScienceDirect y CINAHL, utilizando los descriptores "anciano" (anciano), "acupuntura auricular" (acupuntura auricular O acupuntura auricular) y "reducción del dolor" (dolor ). La búsqueda inicial encontró 179 estudios, se incluyeron artículos originales, disponibles en su totalidad, en cualquier idioma y no se adoptó un marco de tiempo. Después de revisar los títulos y los resúmenes, quedaron 15 para el análisis del texto completo. Finalmente, se incluyeron 11 estudios en la revisión. Resultados: La efectividad de la acupuntura auricular en el control y tratamiento del dolor se evidenció en diferentes poblaciones, como ancianos oncológicos, individuos sometidos a procedimientos quirúrgicos y con algún tipo de dolor. Conclusión: A pesar del número limitado de estudios con ancianos, la diversidad de poblaciones y los diferentes protocolos de intervención, se recomienda que la AA asociada o no a otros tratamientos pueda contribuir a la reducción del dolor en el anciano.

Palabras clave: Anciano; Acupuntura de oído; Reducción del dolor. 


\section{Introdução}

O Brasil tem passado por um processo de mudança demográfica da população. Segundo dados do IBGE, temos em torno de 29 milhões de brasileiros na faixa etária de 60 anos ou mais, o que equivale a 14,3\% da população do país. As projeções indicam que em 2050, a população idosa representará cerca de 30\% do total (IBGE, 2018). Diante disso, com o aumento do percentual de idosos, eleva-se a necessidade de cuidados integrais e singulares decorrentes do contexto e da multidimensionalidade dessa parcela populacional.

O processo de envelhecimento traz consigo diversas particularidades, incluindo alterações nos diferentes sistemas corporais, o que impõe limitações na capacidade funcional e na qualidade de vida do idoso, o que torna esse público mais vulnerável. Ao considerar as alterações funcionais do processo de senescência, com o aumento da prevalência de doenças típicas e das incapacidades associadas, muitas das limitações são impostas pela presença de dores agudas ou crônicas, sejam musculares, ósseas ou articulares, que são explicadas, por exemplo, pela sarcopenia, osteoporose e artrose comuns nessa etapa de vida (SBGG, 2018).

Ademais, além da dor, vivenciada de maneira diferente por cada indivíduo, na fase idosa é possível evidenciar a presença de outros acometimentos em consonância com as alterações comuns do processo de senescência, tais como crônicos: diabetes, hipertensão arterial, insuficiência cardíaca e Alzheimer e, ainda, predispor agudos: pneumonia, infecção de trato urinário e fraturas por quedas etc, o que acarreta consequências na vida do idoso (Castro, et al., 2020).

A dor é tida como a causa mais comum de tratamento médico, com isso, a população idosa recorre ao tratamento farmacológico, em que acabam utilizando múltiplos fármacos, prática conhecida como polifarmácia somado a automedicação. Assim, o abuso excessivo de medicamentos para diferentes problemas de saúde, consequentemente com diferentes composições e ações farmacológicas, sobrecarregam outros sistemas corporais do idoso, reduzindo a função de órgãos, tais como fígado e rins, aumentando o risco de efeitos colaterais e intoxicações, o que leva ao agravamento do estado de saúde e ao surgimento da dor (Freitas \& Py, 2017).

A presença de dor no idoso evidencia algum comprometimento da saúde, como mencionado anteriormente, podendo afetar sua autonomia e independência, principalmente quando esta é carregada durante anos sem a intenção comunicada pelo idoso de investigar a causa. Essa não aceitação do adoecimento é um fator predisponente de agravos, especialmente em pessoas com mais de 60 anos e que vivem sozinhas, predispondo uma associação da síndrome da fragilidade com comprometimentos psicossociais e abuso de medicamentos (em sua maioria anti-inflamatórios e analgésicos) (Castro, et al., 2020). Tendo isso em vista, a adesão das Práticas Integrativas e Complementares em Saúde (PICS), ascende como estratégia interessante na desmedicalização e enfrentamento da dor, angústias e estresses, como métodos não farmacológicos e não invasivos eficazes na melhora da qualidade de vida e no alívio da dor, comprovados cientificamente e com seguridade.

As PICS foram institucionalizadas no SUS no ano de 2006, pela Portaria nº 971, de 03 de maio de 2006 que aprovou a Política Nacional de Práticas Integrativas e Complementares em Saúde (PNPICS), tal política foi implementada e ganhou visibilidade com o intuito de integrar os sistemas médicos complexos e recursos terapêuticos aos sistemas de saúde, buscando estimular mecanismos naturais de prevenção de agravos e recuperação da saúde por meio de ações eficazes e seguras, com ênfase na escuta acolhedora, no desenvolvimento do vínculo terapêutico e na integração do ser humano com o meio e a sociedade, o que contribui positivamente para o efeito da intervenção, contribuindo para promoção da saúde e permitindo com que vários profissionais da área de saúde contribuam e realizem tais práticas de forma gratuita e integral, mantendo uma abordagem ampliada do processo saúde-doença (Brasil, 2020).

A Acupuntura Auricular (AA), como PICS, consiste em uma técnica de intervenção não farmacológica em saúde que faz parte dos recursos terapêuticos da Medicina Tradicional Chinesa (MTC), que utiliza do pavilhão auricular para exercer estímulos que ativa canais de energia em todo o corpo, potencializando o estado de saúde e estabelecendo uma manutenção 
energética, por meio de agulhas, esferas de aço, ouro, prata ou sementes de mostarda. A estimulação por meio da AA tem a capacidade de propiciar a liberação de neurotransmissores e outras substâncias responsáveis pela analgesia, sendo de boa aceitação por aqueles que aderem seu tratamento terapêutico (Azevedo, et al., 2021).

À vista disso, o interesse na realização desta pesquisa originou-se da necessidade da aplicação de práticas alternativas e não farmacológicas, que não venham a trazer prejuízos futuros na saúde do idoso. Nesse contexto, emergiu a seguinte questão: Quais as evidências disponíveis na literatura sobre o uso da acupuntura auricular na redução da dor em idosos?

O cuidado de enfermagem é imprescindível na assistência ao idoso, visto que é uma fase de vida muito delicada, marcada por diversas alterações biopsicossociais. O enfermeiro e sua equipe têm papel em todas as etapas da assistência, do atendimento às necessidades do idoso, na promoção da saúde e na prevenção de agravos. Dessa forma, o uso da acupuntura auricular vem sendo uma ferramenta aplicada para auxiliar no tratamento da dor e visa àmanutenção do equilíbrio físico e mental, consequentemente, a melhora da dor e qualidade de vida.

Visa-se contribuir na formação de profissionais enfermeiros mais capacitados e humanizados ao exercer a prática do cuidado e no desenvolvimento das atividades em saúde, desfrutando-se das PICS, como a acupuntura auricular, principalmente no que se refere à assistência ao idoso. O enfermeiro como integrante da equipe de saúde, necessita cada vez mais de conhecimentos técnico-científicos para prestar uma assistência adequada ao idoso e a sua família. Dessa forma, deve atuar de forma humanizada e integral, usufruindo de novas práticas em saúde, a fim de contribuir na implementação de intervenções e executá-las de forma qualificada, visando melhores respostas ao tratamento e prevenindo agravos.

Este estudo tem como relevância demonstrar a utilização da acupuntura auricular no alívio da dor em idosos, destacando a eficácia desse método para obtenção da melhora na qualidade de vida da pessoa idosa e, assim, reduzir a utilização de medicamentos para a analgesia. Os conhecimentos adquiridos promoverão um benefício múltiplo, aos estudantes e profissionais de saúde, essencialmente os enfermeiros, pois terão um melhor conhecimento sobre as PICS, em especial, sobre a acupuntura auricular na redução da dor no idoso, realizar um atendimento exclusivo, humanizado e integral, atendendo suas necessidades.

Devido ao exposto acima, o presente trabalho tem como objetivo analisar as evidências disponíveis na literatura sobre o uso da acupuntura auricular na redução da dor em idosos.

\section{Metodologia}

Trata-se de uma revisão integrativa da literatura, com abordagem quantitativa, que segundo Mendes, Silveira \& Galvão (2008, p. 760) consiste na "construção de uma análise ampla da literatura, contribuindo para discussões sobre métodos e resultados de pesquisas, bem como reflexões sobre a realização de futuros estudos".

Para conduzir esta investigação, percorreram-se cinco etapas definidas por Whittemore, et al. (2005): elaboração da questão de pesquisa, busca na literatura dos estudos primários, avaliação dos estudos primários, análise dos dados e apresentação da revisão.

Desse modo, utilizou-se a estratégia PICO (JBI, 2021) para a elaboração da questão norteadora: "Quais as evidências disponíveis na literatura sobre o uso da acupuntura auricular na redução da dor em idosos?”. Assim sendo, "P” de população (adultos e idosos), "I" de intervenção (acupuntura auricular), "C" de comparação, não se aplica e, para o elemento "O" (desfecho), foi considerada a redução da dor (Quadro 1). 
Quadro 1. Descrição da estratégia PICO e equação de busca da revisão.

\begin{tabular}{|c|c|c|c|c|}
\hline \multirow[t]{2}{*}{ Pergunta } & \multicolumn{4}{|c|}{$\begin{array}{l}\text { Quais as evidências disponíveis na literatura sobre o uso da acupuntura auricular na redução da dor em } \\
\text { idosos? }\end{array}$} \\
\hline & População (P) & Intervenção (I) & Comparação (C) & $\operatorname{Desfecho}(\mathbf{O})$ \\
\hline Extração & Idosos & Acupuntura auricular & & Redução da dor \\
\hline $\begin{array}{l}\text { Conversão e } \\
\text { Combinação }\end{array}$ & Idoso (Aged) & $\begin{array}{l}\text { Acupuntura auricular } \\
\text { (Auricular Acupuncture) } \\
\text { Acupuntura de Orelha } \\
\text { (Ear Acupuncture) }\end{array}$ & & Dor (Pain) \\
\hline Construção & (Aged) & $\begin{array}{l}\text { ("Auricular } \\
\text { Acupuncture" OR "Ear } \\
\text { Acupuncture") }\end{array}$ & Não se aplica & (Pain) \\
\hline Uso & \multicolumn{4}{|c|}{ (Aged) AND ("Auricular Acupuncture" OR "Ear Acupuncture") AND (Pain) } \\
\hline
\end{tabular}

Fonte: adaptado de Araújo (2020).

A busca foi realizada nas seguintes bases de dados: Medical Literature Analysis and Retrieval System Online (MEDLINE), Literatura Latino-Americana e do Caribe em Ciências da Saúde (LILACS), Web of Science, Scopus, ScienceDirect e Cumulative Index to Nursing and Allied Health Literature (CINAHL).

Para cada base de dados, os descritores controlados foram combinados por meio dos operadores booleanos OR e AND. Foram utilizados os Descritores em Ciências da Saúde (DeCS) e seus correspondentes do Medical Subject Headings (MeSH): idoso (aged), acupuntura auricular (auricular acupuncture OR ear acupuncture) e redução da dor (pain).

As estratégias de buscas para cada base estão descritas no Quadro 2, na qual foram utilizadas as equações de busca no idioma que possibilitou encontrar um maior número de artigos.

Quadro 2. Estratégias de buscas utilizadas nas bases de dados CINAHL, Web of Science, Medline, Scopus, ScienceDirect e LILACS.

\begin{tabular}{|l|l|}
\hline Base & Estratégias de busca \\
\hline CINAHL & TI=("Auricular Acupuncture" OR "Ear Acupuncture") AND ALL=Aged AND ALL=Pain \\
\hline Web of Science & TI=("Auricular Acupuncture" OR "Ear Acupuncture") AND ALL=Aged AND ALL=Pain \\
\hline MEDLINE & ((Aged) AND ("Auricular Acupuncture"[Title] OR "Ear Acupuncture"[Title])) AND (Pain) \\
\hline Scopus & ALL (aged ) AND TITLE ( "Auricular Acupuncture" OR "Ear Acupuncture" ) AND ALL ( pain ) \\
\hline ScienceDirect & TI=("Auricular Acupuncture" OR "Ear Acupuncture") AND ALL=Aged AND ALL=Pain \\
\hline LILACS & (Aged) AND ("Auricular Acupuncture" OR "Ear Acupuncture") AND (Pain) \\
\hline
\end{tabular}

Fonte: Autores (2021).

A busca foi inicialmente realizada no mês de junho e julho de 2021. Já a avaliação e a análise dos resultados ocorreram em agosto de 2021.

Os critérios de inclusão adotados foram: estudos originais, disponíveis na íntegra, em qualquer idioma e em qualquer período, conduzidos em adultos e idosos que tivessem como objeto de investigação o uso da acupuntura auricular na redução 
da dor. Foram excluídos os estudos que envolveram apenas a aplicação de acupuntura sistêmica ou que abordaram diagnóstico auricular e os que não responderam a questão da pesquisa.

Os dados foram coletados por meio de um formulário elaborado pelas pesquisadoras e organizados em um banco de dados do Microsoft Excel com informações sobre identificação dos artigos (autores, ano, periódico, idioma, país de origem), aspectos metodológicos (objetivo, tipo de estudo, nível de evidência, amostra, intervenção, grupo controle, avaliação), desfecho, principais resultados e conclusões.

Os artigos foram classificados quanto ao nível de evidência segundo Fineout-Overholt \& Stillwell (2011) em: nível 1 - revisões sistemáticas ou metanálises de ensaios clínicos randomizados controlados; nível 2 - ensaio clínico randomizado controlado bem delineado; nível 3 - ensaios clínicos bem delineados sem randomização; nível 4 - estudos de coorte e de casocontrole bem delineados; nível 5 - revisão sistemática de estudos descritivos e qualitativos; nível 6 - estudo descritivo ou qualitativo; nível 7 - opinião de autoridades e/ou relatório de comitês de especialistas.

A análise dos dados foi realizada a partir da leitura criteriosa dos artigos e da interpretação dos resultados, que foram sintetizados e discutidos com a literatura. O estudo seguiu as recomendações do checklist Preferred Reporting Items for Systematic Reviews and Meta-Analyses (PRISMA) e o fluxograma PRISMA para a elaboração da revisão (Page, et al, 2021). Os resultados foram apresentados em quadros, demonstrando os objetivos, aspectos metodológicos e desfechos dos artigos selecionados.

\section{Resultados}

Um total de 179 estudos foi encontrado nas buscas eletrônicas. Por estarem duplicados, 58 foram removidos, resultando em 121. Após a revisão de títulos e resumos, 106 artigos foram excluídos, de forma que 15 permaneceram para a análise do texto completo. Por fim, 4 artigos foram excluídos por não responderem a pergunta, restando 11 que foram incluídos na revisão (Figura 1). 
Figura 1. Fluxograma PRISMA do processo de seleção dos artigos da revisão integrativa.

\section{Identificação de estudos por meio das bases de dados}

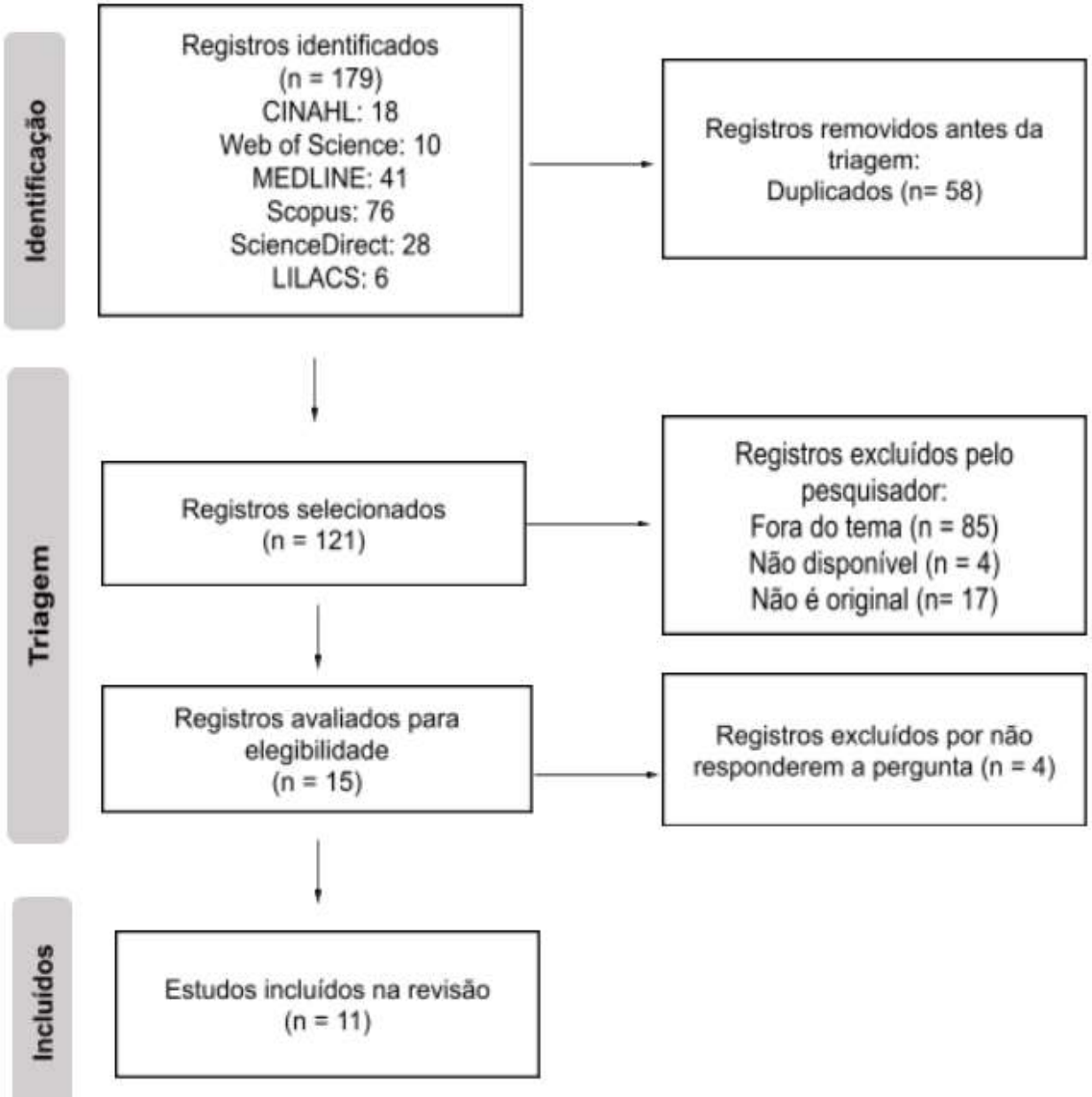

Fonte: Elaborado a partir das recomendações do PRISMA (Page, et al, 2021).

Em relação ao período de publicação, os artigos foram publicados entre 2003 e 2021, com três artigos o ano de 2019 (27\%), dois o ano de 2018 (18\%) e os demais períodos publicaram um artigo cada (9\%). Os artigos foram publicados na língua inglesa $(n=11 ; 73 \%)$ e portuguesa $(n=3 ; 27 \%)$. Os países que mais publicaram foram o Brasil $(n=4 ; 36 \%)$ e os Estados Unidos da América $(n=3 ; 27 \%)$. Também foram encontrados estudos da Áustria $(n=2 ; 18 \%)$, França $(n=1 ; 9 \%)$ e Alemanha $(\mathrm{n}=1 ; 9 \%)$.

Quanto aos periódicos de publicações, os que se destacaram foram periódicos da área da enfermagem $(n=4 ; 36 \%)$. Os demais foram periódicos gerais das ciências da saúde, da área de oncologia e sobre dor ou analgesia $(n=2 ; 18 \%)$. Também foi encontrado um da área de neurologia (9\%). A maioria dos estudos foram ensaios clínicos randomizados com o nível de evidência $2(n=10 ; 91 \%)$. Um estudo foi do tipo coorte com o nível 4 (9\%).

O Quadro 3 apresenta a caracterização dos estudos quanto à identificação, tipo de estudo e nível de evidência, objetivos e desfechos. 
Quadro 3. Caracterização dos estudos selecionados na revisão integrativa quanto ao tipo de estudo, nível de evidência, objetivos e desfechos.

\begin{tabular}{|c|c|c|c|}
\hline Autoria & $\begin{array}{c}\text { Tipo de estudo/ Nível de } \\
\text { evidência }\end{array}$ & Objetivos & Desfechos \\
\hline $\begin{array}{c}\text { Moura et al. } 2018 \\
\text { (A1) }\end{array}$ & $\begin{array}{l}\text { Ensaio clínico } \\
\text { randomizado/nível } 2\end{array}$ & $\begin{array}{l}\text { Avaliar a ação da Auriculoacupuntura (AA) } \\
\text { sobre a incapacidade e a temperatura } \\
\text { tissular em pessoas com dor crônica na } \\
\text { coluna vertebral. }\end{array}$ & $\begin{array}{l}\text { A AA foi eficaz para reduzir a incapacidade e } \\
\text { aumentar a temperatura tissular em pessoas com } \\
\text { dor crônica na coluna vertebral. }\end{array}$ \\
\hline $\begin{array}{c}\text { Ruela et al. } 2018 \\
\text { (A2) }\end{array}$ & $\begin{array}{l}\text { Ensaio clínico } \\
\text { randomizado/nível } 2\end{array}$ & $\begin{array}{l}\text { Avaliar a efetividade da Acupuntura } \\
\text { Auricular na dor de pacientes oncológicos } \\
\text { em tratamento quimioterápico e verificar se } \\
\text { houve alterações no consumo da medicação } \\
\text { analgésica após a aplicação dessa } \\
\text { intervenção. }\end{array}$ & $\begin{array}{l}\text { A AA foi efetiva na redução da intensidade da } \\
\text { dor dos portadores de câncer em tratamento } \\
\text { quimioterápico, além de ter proporcionado a } \\
\text { redução do consumo de analgésicos. }\end{array}$ \\
\hline $\begin{array}{c}\text { Moura et al. } 2019 \\
\text { (A3) }\end{array}$ & $\begin{array}{l}\text { Ensaio clínico } \\
\text { randomizado/nível } 2\end{array}$ & $\begin{array}{l}\text { Comparar a eficácia da acupuntura auricular } \\
\text { chinesa e francesa em pessoas com dor } \\
\text { crônica nas costas. }\end{array}$ & $\begin{array}{l}\text { A intensidade da dor diminuiu } \\
\text { significativamente com a acupuntura auditiva } \\
\text { chinesa. Ambos os tipos de acupuntura auricular } \\
\text { (chinesa e francesa) afetaram a interferência da } \\
\text { dor nas atividades diárias. Porém, na } \\
\text { comparação entre as avaliações inicial e final, } \\
\text { apenas a acupuntura auditiva chinesa apresentou } \\
\text { resultados estatisticamente significativos. }\end{array}$ \\
\hline $\begin{array}{c}\text { Moura et al. } 2019 \\
\text { (A4) }\end{array}$ & $\begin{array}{l}\text { Ensaio clínico } \\
\text { randomizado/nível } 2\end{array}$ & $\begin{array}{l}\text { Avaliar os efeitos da AA chinesa sobre a } \\
\text { intensidade da dor crônica, o alívio } \\
\text { proporcionado pela intervenção, a } \\
\text { interferência da dor nas atividades } \\
\text { cotidianas e o limiar de dor em pessoas com } \\
\text { distúrbios musculoesqueléticos nas costas. }\end{array}$ & $\begin{array}{l}\text { A AA apresentou efeitos positivos ao reduzir a } \\
\text { intensidade da dor crônica e sua interferência nas } \\
\text { atividades cotidianas em pessoas com distúrbios } \\
\text { musculoesqueléticos nas costas. }\end{array}$ \\
\hline $\begin{array}{ll}\text { Usichenko } & \text { et al. } \\
& 2005 \\
& (\mathrm{~A} 5)\end{array}$ & $\begin{array}{l}\text { Ensaio clínico } \\
\text { randomizado/nível } 2\end{array}$ & $\begin{array}{l}\text { Testar se o AA de pontos específicos é } \\
\text { superior à acupuntura sham para analgesia } \\
\text { complementar após artroplastia total de } \\
\text { quadril (ATQ). }\end{array}$ & $\begin{array}{l}\text { Os achados do estudo demonstram que o AA } \\
\text { pode ser usado para reduzir a necessidade de } \\
\text { analgésico pós-operatório. }\end{array}$ \\
\hline $\begin{array}{l}\text { Sator- } \\
\qquad \begin{array}{r}\text { Katze } \\
\text { nschla } \\
\text { ger }\end{array} \\
\text { et al. } 2004 \text { (A6) }\end{array}$ & $\begin{array}{l}\text { Ensaio clínico } \\
\text { randomizado/nível } 2\end{array}$ & $\begin{array}{l}\text { Testar a hipótese de que a estimulação } \\
\text { elétrica dos pontos de acupuntura auricular } \\
\text { (EA) é mais eficaz do que a acupuntura } \\
\text { auricular manual convencional (CO) nesses } \\
\text { pacientes. }\end{array}$ & $\begin{array}{l}\text { O alívio da dor foi significativamente melhor no } \\
\text { grupo EA em comparação com o grupo CO. A } \\
\text { EA reduziu efetivamente as qualidades da dor } \\
\text { neuropática e nociceptiva, como também } \\
\text { melhorou variáveis objetivas, como o consumo } \\
\text { de medicação. Além disso, os pacientes tratados } \\
\text { com EA mostraram uma melhora significativa } \\
\text { no bem-estar psicológico, atividade física e } \\
\text { qualidade do sono e, ainda, voltaram ao trabalho } \\
\text { em tempo integral mais cedo do que os pacientes } \\
\text { tratados com CO como resultado direto do } \\
\text { aumento da qualidade de vida. }\end{array}$ \\
\hline Alimiet al. 2003 & $\begin{array}{l}\text { Ensaio clínico } \\
\text { randomizado/nível } 2\end{array}$ & $\begin{array}{l}\text { Examinar a eficácia da acupuntura auricular } \\
\text { na redução da intensidade da dor em } \\
\text { pacientes com câncer. }\end{array}$ & $\begin{array}{l}\text { A acupuntura auricular foi associada a uma } \\
\text { redução significativa na intensidade da dor para } \\
\text { os pacientes com câncer que estão com dor. A } \\
\text { intensidade da dor diminuiu } 36 \% \text { em } 2 \text { meses da } \\
\text { linha de base no grupo que recebeu acupuntura, } \\
\text { houve pouca mudança para os pacientes que } \\
\text { receberam placebo. }\end{array}$ \\
\hline $\begin{array}{c}\text { Mao et al. } 2021 \\
\text { (A8) }\end{array}$ & $\begin{array}{l}\text { Ensaio clínico } \\
\text { randomizado/ nível } 2\end{array}$ & $\begin{array}{l}\text { Determinar a eficácia da eletroacupuntura } \\
\text { ou acupuntura auricular para dor } \\
\text { musculoesquelética crônica } \\
\text { sobreviventes de câncer. }\end{array}$ & $\begin{array}{l}\text { Entre sobreviventes de câncer com dor } \\
\text { musculoesquelética crônica, tanto a } \\
\text { eletroacupuntura como a acupuntura auricular } \\
\text { produziram maior redução da intensidade da dor } \\
\text { do que o tratamento usual. No entanto, a } \\
\text { acupuntura auricular não demonstrou não } \\
\text { inferioridade à eletroacupuntura, e os pacientes } \\
\text { que a receberam tiveram mais eventos adversos. }\end{array}$ \\
\hline
\end{tabular}




\begin{tabular}{|c|c|c|c|}
\hline $\begin{array}{c}\text { Zeliadtet al. } 2020 \\
\text { (A9) }\end{array}$ & $\begin{array}{l}\text { Estudo de coorte } \\
\text { transversal/nível } 4\end{array}$ & $\begin{array}{l}\text { Avaliar a eficácia da Acupuntura do Campo } \\
\text { de Batalha (BFA) no alívio imediato da dor } \\
\text { e identificar subgrupos de pacientes para os } \\
\text { quais a BFA é mais eficaz. }\end{array}$ & $\begin{array}{l}\text { O BFA foi eficaz para muitos veteranos com dor } \\
\text { crônica preexistente ou com comorbidades } \\
\text { físicas e psicológicas. Os veteranos com uso de } \\
\text { opióides no ano anterior ao BFA } \\
\text { experimentaram menos melhora, com os escores } \\
\text { de intensidade da dor melhorando mais entre os } \\
\text { veteranos que não usaram opióides } \\
\text { recentemente. Os veteranos relataram que o BFA } \\
\text { é uma intervenção eficaz e segura para diminuir } \\
\text { a intensidade da dor de curto prazo. }\end{array}$ \\
\hline $\begin{array}{l}\text { Krusche-Mandl } \\
\text { et al. } 2019 \text { (A10) }\end{array}$ & $\begin{array}{l}\text { Ensaio piloto } \\
\text { randomizado/nível } 2\end{array}$ & $\begin{array}{l}\text { Analisar se a acupuntura auricular elétrica } \\
\text { (EAA) ou a terapia por corrente elétrica } \\
\text { modulada de baixa frequência (LFMECT) } \\
\text { podem apoiar o tratamento analgésico. }\end{array}$ & $\begin{array}{l}\text { A implementação de EAA ou LFMECT parece } \\
\text { ser benéfica para reduzir a dor no joelho e } \\
\text { melhorar a função do joelho em pacientes com } \\
\text { osteoartrite de joelho. Além disso, ambas } \\
\text { mostraram um efeito contínuo e podem ser uma } \\
\text { modalidade de tratamento adicional, em } \\
\text { particular para pacientes com comorbidades } \\
\text { médicas graves que apresentam alto risco de } \\
\text { complicações na cirurgia de substituição total da } \\
\text { articulação. }\end{array}$ \\
\hline $\begin{array}{r}\text { Estores et al. } 2017 \\
\text { (A11) }\end{array}$ & $\begin{array}{l}\text { Ensaio clínico piloto } \\
\text { randomizado/nível } 2\end{array}$ & $\begin{array}{l}\text { Obter dados preliminares sobre os efeitos } \\
\text { de um protocolo de acupuntura auricular, } \\
\text { Battlefield Acupuncture (BFA), na } \\
\text { intensidade da dor autorreferida em pessoas } \\
\text { com Lesão Medular Crônica (LM) e dor } \\
\text { neuropática. }\end{array}$ & $\begin{array}{l}\text { A acupuntura auricular com agulhas } \\
\text { semipermanentes em pontos específicos (BFA), } \\
\text { fornecida semanalmente a pacientes com dor } \\
\text { neuropática abaixo do nível devido a uma lesão } \\
\text { medular crônica, proporcionou redução } \\
\text { clinicamente significativa na intensidade da dor. }\end{array}$ \\
\hline
\end{tabular}

Fonte: Autores (2021).

As amostras dos estudos clínicos foram entre 23 e 360 adultos e idosos com idades entre 18 e 84 anos. O estudo coorte avaliou 11.406 prontuários de pacientes entre 18 e mais de 80 anos. Não foram encontrados estudos apenas com idosos e que abordavam as particularidades do envelhecimento. O tipo de dor mais estudado foi a dor musculoesquelética (n= 7; 64\%) em diversas regiões, por exemplo, dor nas costas, dor lombar e dor no joelho. Também foram estudadas a dor oncológica (n= 2; 18\%), dor pós-artroplastia total do quadril e dor neuropática relacionada à lesão da medula espinhal (n=1; $9 \%$ cada).

As intervenções realizadas foram Acupuntura Auricular (AA) seguindo a Medicina Tradicional Chinesa (A1, A2, A3, A4), AA Francesa (A3), Acupuntura elétrica (A8, A9, A10), Acupuntura do Campo de Batalha (BFA) (A9, A11) e AA seguindo outros protocolos e relatórios clínicos (A5, A7). Quanto aos grupos controles, alguns participantes não receberam nenhuma intervenção (A1, A3, A4, A11) ou receberam AAem pontos placebos não relacionados com a dor (A1, A2, A5, A7). Em um artigo (A8), o grupo controle recebeu cuidados usuais para o tratamento da dor, como analgésicos e fisioterapia.

As características relacionadas ao protocolo de intervenção foram descritas no Quadro 4. 
Quadro 4. Caracterização dos estudos selecionados na revisão integrativa quanto a intervenção aplicada, pontos, material, sessões e ferramentas de avaliação.

\begin{tabular}{|c|c|c|c|c|c|}
\hline Artigo & Intervenção & Pontos & Material & Sessões & Avaliação \\
\hline A1 & $\begin{array}{l}\text { AA de acordo com o } \\
\text { equilíbrio energético e } \\
\text { segundo os padrões da } \\
\text { Medicina Tradicional } \\
\text { Chinesa } \\
\text { Seguiu-se } \\
\text { recomendações as } \\
\text { Standards do } \\
\text { Reporting Interventions } \\
\text { in Clinical Trials of } \\
\text { Acupuncture } \\
\text { (STRICTA) }\end{array}$ & $\begin{array}{lll}\text { Shenmen } & \text { (TF4); Rim } & \text { (CO10); } \\
\text { Nervo } & \text { Simpático } & \text { (AH6a); } \\
\text { Vértebras Cervicais (HA13), } & \text { Córtebras Torácicas (AH11) e/ou } \\
\text { Vértebras Lombares (AH9) }\end{array}$ & $\begin{array}{l}\text { Agulhas auriculares } \\
\text { estéreis, descartáveis } \\
\text { semipermanentes }\end{array}$ & $\begin{array}{l}\text { Cinco sessões } \\
\text { uma vez por } \\
\text { semana, durante } \\
\text { um mês e meio, } \\
\text { com alternância } \\
\text { do pavilhão } \\
\text { auricular a cada } \\
\text { sessão }\end{array}$ & $\begin{array}{l}\text { Avaliação da interferência } \\
\text { da dor nas atividades de } \\
\text { vida diária pelo } \\
\text { Questionário de } \\
\text { Incapacidade de Rolland } \\
\text { Morris (QIRM) }\end{array}$ \\
\hline A2 & $\begin{array}{l}\text { Acupuntura Auricular } \\
\text { em pontos do equilíbrio } \\
\text { energético seguindo a } \\
\text { teoria dos Cinco } \\
\text { Elementos da MTC e } \\
\text { em pontos indicados } \\
\text { para o tratamento da } \\
\text { dor }\end{array}$ & $\begin{array}{l}\text { Pontos Shenmen, Rim, Simpático, } \\
\text { Relaxamento Muscular e os } \\
\text { pontos do equilíbrio energético, } \\
\text { definidos por meio da teoria dos } \\
\text { Cinco Elementos }\end{array}$ & $\begin{array}{l}\text { Agulhas auriculares } \\
\text { estéreis, descartáveis } \\
\text { semipermanentes, } \\
\text { tamanho } 0,20 \mathrm{~mm} \mathrm{x} \\
1,5 \mathrm{~mm}\end{array}$ & $\begin{array}{lr}\text { Oito sessões de } \\
\text { AA uma vez por } \\
\text { semana, com } \\
\text { intervalo de } 7 \\
\text { dias entre as } \\
\text { sessões, } \\
\text { alternando } \\
\text { pavilhão } \\
\text { auricular a cada } \\
\text { sessão. } & \text { Tempo } \\
\text { médio } & \text { por } \\
\text { sessão: } & 40 \\
\text { minutos } & \end{array}$ & $\begin{array}{l}\text { Avaliação da intensidade } \\
\text { da dor pela Escala } \\
\text { Numérica da Dor (EN), na } \\
\text { versão de } 11 \text { pontos. } \\
\text { Avaliação do consumo de } \\
\text { analgésicos por meio do } \\
\text { relato dos participantes } \\
\text { sobre doses diárias, número } \\
\text { de analgésicos e } \\
\text { enquadramento no degrau } \\
\text { da Escada Analgésica da } \\
\text { Organização Mundial da } \\
\text { Saúde }\end{array}$ \\
\hline A3 & $\begin{array}{lr}\text { Acupuntura } & \text { Auricular } \\
\text { chinesa e } & \text { francesa. } \\
\text { Seguiu-se } & \text { as } \\
\text { recomendações } & \text { do } \\
\text { STRICT } & \end{array}$ & $\begin{array}{l}\text { AA chinesa: Shenmen (TF4), } \\
\text { Rim (CO10) e Sistema Nervoso } \\
\text { Simpático (AH6a), pontos de } \\
\text { equilíbrio energético } \\
\text { correspondendo a um par de um } \\
\text { órgão e uma víscera (Coração - } \\
\text { CO15 e Intestino delgado - CO6 / } \\
\text { Rim - CO10 e Bexiga - CO9 / } \\
\text { Fígado - CO12 e Vesícula Biliar - } \\
\text { CO11 / Pulmão - CO14 e } \\
\text { Intestino grosso - CO7 / Baço - } \\
\text { CO13 e Estômago - CO4) } \\
\text { seguidos por Vértebras Cervicais } \\
\text { (HA13), Vértebras Torácicas } \\
\text { (AH11) e / ou Vértebras } \\
\text { Lombares (AH9), dependendo do } \\
\text { local da queixa de dor. } \\
\text { AA francesa: cinco pontos fixos } \\
\text { na mesma ordem: Zero, Coluna } \\
\text { Cervical, Coluna Torácica, } \\
\text { Coluna Lombar e Síntese. }\end{array}$ & $\begin{array}{l}\text { Agulhas auriculares } \\
\text { semipermanentes, } \\
\text { estéreis } \\
\text { descartáveis, } \\
\text { tamanho } 0,20 \times 1,5 \\
\text { mm. } \\
\text { Auxílio de um } \\
\text { localizador de pontos } \\
\text { de acupuntura } \\
\text { (DongBang, Acu - } \\
\text { Treat@) }\end{array}$ & $\begin{array}{l}\text { Cinco sessões } \\
\text { individuais, uma } \\
\text { vez por semana, } \\
\text { por um mês e } \\
\text { meio, rom } \\
\text { alternância de } \\
\text { orelha a cada } \\
\text { sessão }\end{array}$ & $\begin{array}{l}\text { Intensidade da dor pelo } \\
\text { Inventário Breve de Dor } \\
\text { (BPI). Interferência da dor } \\
\text { nas atividades de vida } \\
\text { diária pelo } \\
\text { Questionário de } \\
\text { Incapacidade de Rolland } \\
\text { Morris (QIRM). Avaliação } \\
\text { da temperatura por uma } \\
\text { câmera termográfica }\end{array}$ \\
\hline A4 & $\begin{array}{l}\text { Auriculoacupuntura } \\
\text { com base no equilíbrio } \\
\text { energético segundo os } \\
\text { padrões da MTC e } \\
\text { seguindo } \\
\text { recomendações as } \\
\text { STRICT }\end{array}$ & $\begin{array}{l}\text { Shenmen } \\
\text { Sistema); Rim } \\
\text { (CO10); } \\
\text { (AH6a); Nervoso Simpático } \\
\text { restabelecimento do equilíbrio } \\
\text { energético, correspondente a um } \\
\text { órgão e uma víscera; Vértebras } \\
\text { Cervicais (AH13), Vértebras } \\
\text { Torácicas (AH11) e/ou Vértebras } \\
\text { Lombossacrais (AH9) }\end{array}$ & $\begin{array}{l}\text { Agulhas auriculares } \\
\text { semipermanentes, } \\
\text { esterilizadas } \\
\text { descartáveis, } \\
\text { tamanho 0,20 x } 1,5 \\
\text { milímetros. } \\
\begin{array}{l}\text { Localizador de } \\
\text { pontos Acu-Treat } \\
\text { (DongBang@) }\end{array}\end{array}$ & $\begin{array}{l}\text { Cinco sessões } \\
\text { uma vez por } \\
\text { semana, durante } \\
\text { um mês e meio, } \\
\text { com alternância } \\
\text { do pavilhão } \\
\text { auricular a cada } \\
\text { sessão }\end{array}$ & $\begin{array}{l}\text { Intensidade da dor pelo } \\
\text { Inventário Breve de Dor } \\
\text { (BPI) e a interferência da } \\
\text { dor nas atividades } \\
\text { cotidianas, o } r \text { alívio } \\
\text { proporcionado r pela } \\
\text { intervenção e o limiar de } \\
\text { dor pela algometria digital }\end{array}$ \\
\hline
\end{tabular}




\begin{tabular}{|c|c|c|c|c|c|}
\hline A5 & 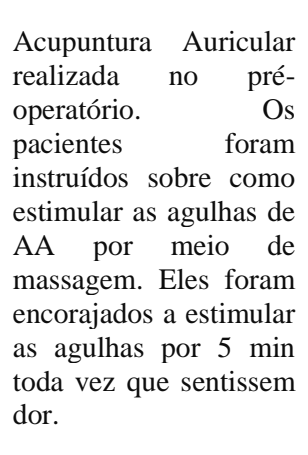 & $\begin{array}{l}\text { Quatro pontos específicos de } \\
\text { acupuntura ipsilateral ao local da } \\
\text { cirurgia: articulação do quadril, } \\
\text { shenmen, pulmão e tálamo. Foi } \\
\text { seguido o protocolo de Oleson } \\
\text { (1998) e outros relatórios } \\
\text { clínicos. }\end{array}$ & $\begin{array}{l}\text { Agulhas descartáveis } \\
\text { de aço com diâmetro } \\
\text { de } 0,22 \mathrm{~mm} \text { e o } \\
\text { comprimento de } 1,5 \\
\text { mm }\end{array}$ & $\begin{array}{l}\text { As agulhas foram } \\
\text { inseridas na noite } \\
\text { anterior à } \\
\text { cirurgia, fixadas } \\
\text { com fita adesiva } \\
\text { da cor da pele e } \\
\text { retidas in situ } \\
\text { durante os } 3 \text { dias } \\
\text { após a cirurgia. }\end{array}$ & $\begin{array}{l}\text { Intensidade da dor pela } \\
\text { Escala Visual Analógica } \\
\text { (VAS) de } 100 \text { mm. } \\
\text { Avaliação da necessidade } \\
\text { de fentanil no } \\
\text { intraoperatório, } \\
\text { temperatura corporal, } \\
\text { frequência cardíaca, } \\
\text { pressão arterial, contagem } \\
\text { de leucócitos, velocidade } \\
\text { de hemossedimentação e a } \\
\text { atividade da proteína C } \\
\text { reativa. }\end{array}$ \\
\hline A6 & $\begin{array}{l}\text { Acupuntura auricular } \\
\text { manual convencional e } \\
\text { estimulação elétrica } \\
\text { dos pontos de } \\
\text { acupuntura auricular. }\end{array}$ & $\begin{array}{l}\text { Agulhas foram inseridas no lado } \\
\text { dominante os seguintes pontos de } \\
\text { acupuntura: coluna lombar, 40; } \\
\text { shenmen, 55; e almofada, 29. Os } \\
\text { pontos foram identificados } \\
\text { medindo-se a resistência da pele } \\
\text { por meio de um medidor de } \\
\text { condutância elétrica (multipoint } \\
\text { selection pen TM, Biegler GmbH, } \\
\text { Mauerbach, Áustria). }\end{array}$ & $\begin{array}{l}\text { Agulhas de } \\
\text { acupuntura } \\
\text { descartáveis, calibre } \\
27,3 \text { mm de } \\
\text { comprimento, } \\
\text { conectadas a um } \\
\text { estimulador } \\
\text { miniaturizado } \\
\text { movido a bateria } \\
\text { recentemente } \\
\text { desenvolvido, usado } \\
\text { atrás da orelha. }\end{array}$ & $\begin{array}{lr}\text { As agulhas } & \text { de } \\
\text { acupuntura } & \text { com } \\
\text { os dispositivos P- } & \text { TM } \\
\text { Stim } \text { Toram } \\
\text { retiradas } & 48 \\
\text { horas após } & \text { a } \\
\text { inserção } & \text { em } \\
\text { todos os casos e } \\
\text { o tratamento de } \\
\text { acupuntura } r \text { foi } \\
\text { realizado uma } \\
\text { vez por semana } \\
\text { por } 48 \text { horas em } \\
\text { casa, por um } \\
\text { período total de } \\
\text { estudo de } & 6 \\
\text { semanas. } & \end{array}$ & $\begin{array}{l}\text { Questionários avaliando a } \\
\text { intensidade e qualidade da } \\
\text { dor, bem-estar psicológico, } \\
\text { nível de atividade e } \\
\text { qualidade do sono, uso de } \\
\text { analgésicos e satisfação } \\
\text { geral com o tratamento. } \\
\text { Questionário de dor McGill } \\
\text { para avaliar a experiência } \\
\text { subjetiva da dor. }\end{array}$ \\
\hline A7 & $\begin{array}{l}\text { Acupuntura auricular } \\
\text { em pontos onde uma } \\
\text { resposta elétrica foi } \\
\text { detectada. }\end{array}$ & $\begin{array}{l}\text { Os pontos foram identificados } \\
\text { pela deteção de um sinal } \\
\text { elétrico. Um gráfico elétrico da } \\
\text { orelha foi estabelecido para cada } \\
\text { paciente medindo-se a resposta } \\
\text { eletrodérmica nos pontos onde } \\
\text { havia suspeita de dor projetada, } \\
\text { com base nos sintomas clínicos. }\end{array}$ & $\begin{array}{l}\text { Implantes de aço em } \\
\text { ponta lança com } 3,4 \\
\text { mm de comprimento } \\
\text { e cabeça cilíndrica } \\
\text { com } 1,2 \text { mm de } \\
\text { diâmetro e altura. } \\
\text { Sementes auriculares } \\
\text { para o grupo placebo }\end{array}$ & $\begin{array}{l}\text { Dois cursos de } \\
\text { tratamento com } \\
\text { intervalo de um } \\
\text { mês, os pacientes } \\
\text { foram solicitados } \\
\text { a relatar as datas } \\
\text { em que as } \\
\text { agulhas ou } \\
\text { sementes caíram. }\end{array}$ & $\begin{array}{l}\text { Avaliação da intensidade } \\
\text { da dor Escala Visual } \\
\text { Analógica (VAS) e do } \\
\text { consumo de analgésicos. }\end{array}$ \\
\hline A8 & $\begin{array}{l}\text { Eletroacupuntura, } \\
\text { acupuntura auricular e } \\
\text { cuidados habituais. }\end{array}$ & $\begin{array}{l}\text { Pontos do giro cingulado, tálamo, } \\
\text { ômega } 2 \text {, ponto zero e shenmen. }\end{array}$ & $\begin{array}{l}\text { Agulha } \\
\text { semipermanentes } \\
\text { (Auguille } \\
\text { Semipermanent } \\
\text { ASP) (Sedatelec) }\end{array}$ & $\begin{array}{lrr}\text { Os } & \text { grupos } & \text { de } \\
\text { intervenção } & \\
\text { receberam } & 10 \\
\text { sessões } & \text { semanais } \\
\text { de } & & \\
\text { eletroacupuntura } \\
\text { e } & \text { acupuntura } \\
\text { auricular. } & \text { Dez } \\
\text { sessões } & \text { de } \\
\text { acupuntura } & \\
\text { foram oferecidas } \\
\text { ao grupo de } \\
\text { cuidados rr das } \\
\text { habituais r dan } \\
\text { semanas } 12 \text { a } 24 .\end{array}$ & $\begin{array}{l}\text { Escala do Inventário Breve } \\
\text { da Dor (BPI) para avaliar a } \\
\text { intensidade da dor e } \\
\text { interferência funcional } \\
\text { relacionada à dor. }\end{array}$ \\
\hline A9 & $\begin{array}{l}\text { Dados de prontuário } \\
\text { eletrônicos } \\
\text { veteranos de guerra } \\
\text { tratados } \\
\text { Acupuntura do Campo } \\
\text { de Batalha (BFA) }\end{array}$ & $\begin{array}{l}\text { Definição da orelha dominante } \\
\text { através da colocação de uma } \\
\text { agulha no giro cingulado da } \\
\text { orelha que apresentar atenuação } \\
\text { do nível da dor. Ponto do tálamo, } \\
\text { ponto ômega 2, ponto zero e no } \\
\text { ponto Shenmen. }\end{array}$ & Não descreve & Não descreve & $\begin{array}{l}\text { Registro do nível de } \\
\text { intensidade de dor } \\
\text { imediatamente antes e após } \\
\text { o procedimento BFA com a } \\
\text { Defense and Veterans Pain } \\
\text { Rating Scale (DVPRS). }\end{array}$ \\
\hline
\end{tabular}




\begin{tabular}{|c|c|c|c|c|c|}
\hline A10 & 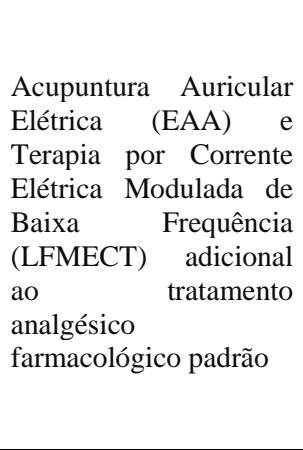 & $\begin{array}{l}\text { Grupo de EAA: Shenmen 55, } \\
\text { joelho } 49 \text { e almofada } 29 . \\
\text { Grupo de LFMECT: pontos } \\
\text { meridianos de acupuntura do } \\
\text { corpo, o meridiano da bexiga e os } \\
\text { pontos de Bachmann estimulados } \\
\text { com o dispositivo SCENAR }{ }^{\circledR} \text { TM }\end{array}$ & $\begin{array}{l}\text { Agulhas de } \\
\text { acupuntura } \\
\text { descartáveis, } \\
\text { dispositivo P-Stim }{ }^{\circledR} \\
\text { TM, aparelho } \\
\text { SCENAR } ® \text { TM }\end{array}$ & $\begin{array}{lr}\text { O estudo foi } \\
\text { realizado por } 42 \\
\text { dias, com as } \\
\text { sessões r de } \\
\text { acupuntura nos } \\
\text { dias } 1,7,14,21, \\
28 \text { e } 35 .\end{array}$ & $\begin{array}{l}\text { Os participantes } \\
\text { registraram o consumo de } \\
\text { medicamentos, } \\
\text { intensidade da dor pela } \\
\text { Escala de Avaliação } \\
\text { Numérica (NRS), bem- } \\
\text { estar psicológico, qualidade } \\
\text { do sono, náuseas, vômitos e } \\
\text { fadiga. Também foi } \\
\text { realizada avaliação clínica } \\
\text { do derrame no joelho e da } \\
\text { amplitude de movimento } \\
\text { ativa. }\end{array}$ \\
\hline A11 & $\begin{array}{l}\text { Acupuntura auricular } \\
\text { baseada no protocolo } \\
\text { de Acupuntura do } \\
\text { Campo de Batalha } \\
\text { (BFA) adaptado. }\end{array}$ & $\begin{array}{l}\text { Cingulado anterior, tálamo, } \\
\text { ômega-2, Shenmen e ponto zero. }\end{array}$ & $\begin{array}{l}\text { Agulhas } \\
\text { semipermanentes } \\
\text { (ASP) estéreis de } \\
\text { ouro }\end{array}$ & $\begin{array}{l}\text { Sessão com dez } \\
\text { agulhas uma vez } \\
\text { por semana, } \\
\text { durante oito } \\
\text { semanas, } \\
\text { realizado em } \\
\text { ambas as orelhas }\end{array}$ & $\begin{array}{ll}\text { Avaliação da dor pela } \\
\text { Escala de Avaliação } \\
\text { Numérica (NRS) }\end{array}$ \\
\hline
\end{tabular}

Fonte: Autores (2021).

O ponto mais utilizado foi o Shenmen (A1, A2, A3, A4, A5, A6, A8, A9, A10).Os materiais mais utilizados foram agulhas auriculares semipermanentes (A1, A2, A3, A4, A8, A11), agulhas de aço (A5, A7), agulhas conectadas ao dispositivo P-Stim ${ }^{\mathrm{TM}}$ para estimulação elétrica (A6, A10) e ao dispositivo SCENAR ${ }^{\mathrm{TM}}$ (A10) e sementes auriculares (A7). Para localizar os pontos foi utilizado os aparelhos Acu-Treat (DongBang®) (A3, A4) e o medidor de condutância elétrica (multipoint selection pen $\left.{ }^{\mathrm{TM}}\right)(\mathrm{A} 6)$.

Os instrumentos utilizados para avaliar a dor foram: Escala de Avaliação Numérica (NRS) (A2, A10, A11), Inventário Breve de Dor (BPI) (A3, A4, A8), Escala Visual Analógica (VAS) (A5, A7), Questionário de dor McGill (A6), Questionário de Incapacidade de Rolland Morris (QIRM) (A1, A3) e Defense and Veterans Pain Rating Scale (DVPRS) (A9).

O desfecho mais avaliado foi a redução da intensidade da dor (A2, A3, A4, A6, A7, A8, A9, A10, A11), destacando que a acupuntura auricular foi eficaz para a diminuição da dor de diversos tipos, como a dor musculoesquelética, dor pósoperatória, dor oncológica e dor neuropática. Além disso, foi avaliado a redução da incapacidade e interferência da dor nas atividades diárias (A1, A3, A4). Outros desfechos como o aumento da temperatura tissular (A1) e a redução do consumo de analgésicos (A2, A5, A6) também foram avaliados e obtiveram um resultado significativo do desfecho analisado. Um estudo também encontrou que o uso da AA promoveu bem-estar psicológico, atividade física, qualidade do sono e o retorno ao trabalho em tempo (A6).

Os resultados dos estudos, portanto, revelam que o uso da Acupuntura Auricular tem-se mostrado eficaz no controle e tratamento da dor, além de permitir a melhora da qualidade de vida.

\section{Discussão}

Apesar do limitado número de estudos primários incluídos e das diversificadas populações em estudo, sugere-se que a acupuntura auricular associada ou não a outras terapias complementares pode contribuir para redução da dor em idosos. Diante da análise dos artigos incluídos na revisão, sugere-se que o uso da Acupuntura Auricular foi eficaz na redução da intensidade da dor, na diminuição da incapacidade física e na redução do consumo de medicamentos. Observou-se que não existe um protocolo de AA padronizado entre os estudos, sendo variável o número de sessões, duração do tratamento, dispositivos e pontos de aplicação. O método mais utilizado foi a AA seguindo a Medicina Tradicional Chinesa, com agulhas auriculares semipermanentes, destacando-se o ponto Shenmen. Os principais instrumentos utilizados para a avaliação foram a Escala de Avaliação Numérica (NRS) e o Inventário Breve de Dor (BPI). 
Uma revisão sistemática e metanálise, com 930 participantes dos estudos entre 18 e 90 anos, encontrou uma redução significativa do escore de intensidade da dor crônica nas costas em pacientes submetidos ao tratamento de AA, em relação ao grupo controle. Logo, a AA é uma prática promissora para o tratamento da dor crônica nas costas e para implementação na prática clínica (Moura, et al., 2019).

Outra revisão sistemática e metanálise demonstrou que a AA pode ser eficaz no alívio da dor em 48 horas e apresenta baixo perfil de efeitos colaterais. Por ser uma modalidade rápida e acessível, é promissora para os profissionais da saúde considerarem o seu uso no ambiente ambulatorial, intra-operatório e emergência para a redução da dor (Murakami, Fox \& Dijkers, 2017).

Uma outra aplicabilidade da AA encontrada foi no cuidado com pacientes oncológicos para alívio da dor e melhora dos sintomas relacionados ao uso de quimioterápicos. A AA é recomendada como uma intervenção de enfermagem segura, bem tolerada e com poucos efeitos colaterais. O enfermeiro oncologista pode aumentar a variedade de opções e utilizar diferentes técnicas para melhorar a qualidade de vida dos pacientes, visando favorecer a recuperação e a manutenção da saúde (Contim, Santo \& Moretto, 2019).

A abordagem mais utilizada foi a MTC, que parte do princípio de que a estimulação de pontos de acupuntura ocasionará um efeito positivo na dor, pois os meridianos estão sendo ativados (Murakami, Fox \& Dijkers, 2017). Os meridianos são passagens para o fluxo de "qi" e "sangue", dois fluidos corporais básicos na MTC. Os meridianos se espalham pela superfície de todo o corpo, integrando o interior com o exterior corporal (Wang, Ayati \& Zhang, 2010).

A aplicação do ponto Shenmen foi favorável para o alívio da dor nos artigos selecionados. O ponto está localizado no vértice do ângulo formado pelo ramo superior e inferior da antélice. Atua na ansiedade, estabilização emocional e possui uma ação analgésica e anti-inflamatória em condições de dor (Artioli, Tavares, \& Bertolini, 2019). Outras revisões encontraram que o ponto Shenmen e os pontos reflexos correspondentes à região afetada são os mais utilizados na prática para o alívio da dor (Artioli, Tavares, \& Bertolini, 2019; Moura, et al., 2019). Também é recomendado o uso de um detector elétrico de acupontos para auxiliar na localização do ponto de acordo com a queixa do paciente (Moura, et al., 2019).

O material mais utilizado foram as agulhas semipermanentes, um tipo de material que realiza o próprio estímulo, sem necessitar de estimulação manual. Quando usado sementes, essa estimulação manual é necessária e, quando usado agulhas auriculares, esse estímulo pode ser manual ou elétrico. Um estudo comparou o efeito da eletroacupuntura e da acupuntura auricular convencional para a redução da dor musculoesquelética crônica, foi encontrado que a convencional não demonstrou inferioridade à eletroacupuntura, mas os pacientes tiveram mais eventos adversos (Mao, et al., 2021).

Em relação aos grupos controles, em alguns artigos não foi realizada intervenção e em outros a AA foi realizada em pontos placebos. Também foi utilizado dois grupos controles, um com inserção de agulhas e outro sem inserção de agulhas, para testar o efeito de uma acupuntura não eficaz, em que as agulhas são inseridas em pontos que não são pontos de acupuntura (Alimi, et al., 2003). Em ensaios clínicos de acupuntura, o grupo controle ideal não foi determinado, mas comparar esse grupo com a terapia padrão-ouro ou nenhum procedimento é o melhor modo de avaliar os efeitos da intervenção (Moura, et al., 2019).

Algumas teorias fundamentam os estudos sobre os benefícios da AA na redução significativa da dor, com ação analgésica. Dentre estas, a ativação de fibras nociceptivas do tipo $\mathrm{A} \beta$ (localizadoras) e fibras do tipo $\mathrm{C}$ (de condução lenta e relacionadas a sensações afetivas e motivacionais), aliado a crença experienciada pelo paciente quanto ao toque terapêutico (presente no momento da inserção das agulhas em pontos específicos); a "Teoria do Portão de Controle da Dor", explica que, qualquer dor, seja ela aguda ou crônica, estimula as fibras nervosas $\mathrm{A} \beta$ e $\mathrm{C}$, que são responsáveis por levarem estímulos nervosos da dor até o cérebro, podendo ser interrompido por meio da eletroestimulação; o controle inibitório descendente, que 
por sua vez, possuem componente para supressão da dor, sendo uma característica do Sistema Nervoso Central, para controlar a percepção dolorosa; e as expectativas do paciente em relação ao alívio da dor (Moura, et al., 2019).

Além de avaliar a intensidade da dor, os estudos incluídos analisaram outros aspectos relacionados, como o consumo de analgésicos, bem-estar psicológico, atividade física, qualidade do sono e interferência nas atividades diárias. Realizar uma avaliação abrangente e investigar esses desfechos é relevante, pois diversas dimensões podem ser afetadas pela dor, seja fisiológica, psíquica, social ou espiritual (Moura, et al., 2017).

Evidências científicas têm mostrado os benefícios das PICS integradas à medicina convencional, evidenciando o bemestar, melhora da autoestima, alívio da dor, da insônia e da ansiedade, aumento da disposição, diminuição de sintomas de doenças, além da redução do consumo de medicamentos (Azevedo, et al., 2015). A principal contribuição que este estudo buscou trazer para o campo da pesquisa científica foi evidenciar os benefícios da acupuntura auricular para a pessoa idosa, como uma prática simples, rápida, segura e de baixo custo capaz de contribuir para melhoria da qualidade de vida de diversos pacientes, inclusive de idosos, reduzindo níveis de dor, por possuir efeito analgésico e atuando no controle do estresse, promovendo saúde e prevenindo complicações.

Oliveira, et al. (2011) levanta importantes melhorias na aplicação precoce de acupuntura auricular no início de sintomas decorrentes do envelhecimento. No estudo, é exposto entrevistas com idosos que apresentavam dores osteoarticulares, dores musculares, escoliose e outras deformidades posturais, disfunções intestinais, acometimentos oriundos de fraturas por queda e até humor deprimido, que aceitaram receber sessões de acupuntura durante um determinado tempo. A maioria dos entrevistados relatou uma melhora significativa no alívio de dores, na melhora postural, na atividade intestinal e do trato urinário, no desenvolvimento das Atividades Básicas da Vida Diária (BVD) e das Atividades Instrumentais da Vida Diária (AIVD), o que promoveu uma mudança significativa na qualidade de vida, com melhora do sono, do humor e das interações sociais.

Alguns outros estudos, como o de Suen, et al. (2007), fortalecem o abordado por outros autores antes mencionados, principalmente no que se refere a presença de dor em região lombar de pacientes idosos, sendo um dos achados mais comuns nessa faixa etária e tendo como consequências a depressão, piora da qualidade de vida e redução funcional. A melhor maneira de auxiliar o paciente com tais dores é agir na melhora da circulação sanguínea, relaxando a musculatura e ativando o sangue, já que não existe cura para essa dor. Sabendo que ensaios clínicos e metanálises evidenciam a relação entre os pontos auriculares e respostas por todo o corpo, incluindo órgãos internos e corrente sanguínea, a estimulação desses pontos favorece o alívio da dor lombar, além de demais sofrimentos sistêmicos oriundos do processo de envelhecimento e, algumas vezes, exacerbados por um estilo de vida de exageros e descuidos.

Apesar de que, em alguns dos estudos incluídos, não tenha sido possível avaliar o efeito da acupuntura auricular de forma uniformizada, pois estavam associados a diferentes estímulos (manual e elétrico) e padrões. Decidimos por manter tais investigações devido ao número escasso de publicações na temática e, ao mesmo tempo, da importância e relevância de conhecer os resultados obtidos. Assim, a realização de pesquisas experimentais que tenham por objetivo investigar o efeito da acupuntura auricular, seja como intervenção única ou potencializando algum outro tratamento, torna-se necessária para comprovação dos benefícios desta prática.

Os artigos selecionados para este estudo envolveram adultos e idosos, visto que se observou uma carência de artigos que abordam a acupuntura auricular especificamente em idosos, o que indicou limitações quanto à quantidade de produções científicas que tratam sobre o uso da acupuntura auricular na redução da dor em idosos e pela heterogeneidade dos estudos, além de que, notou-se variações na aplicação da acupuntura auricular, especialmente em relação ao dispositivo de aplicação, a população diversificada e as diferenças de grupo-controle. Também, referente às divergências em relação ao número de sessões, a duração de cada tratamento, o tempo de permanência do dispositivo, o tipo de estímulo, os pontos de aplicação, a 
localização dos pontos e a experiência do profissional que podem influenciar os efeitos clínicos, o que intensifica a necessidade da padronização desta terapia. O uso de protocolos fixos favorece a replicabilidade e a implementação na prática clínica (Moura, et al., 2019).

Um ponto relevante que foi pouco abordado nos artigos é sobre a formação do profissional que aplicou a acupuntura. A acupuntura representa uma terapêutica complexa que busca a saúde integral do indivíduo, o seu desconhecimento propicia uma prática inadequada e excessivamente medicalizada (Nunes, et al., 2017).

Desse modo, é importante que os profissionais da saúde busquem informações sobre outras possibilidades de intervenções e ampliem as formas de tratamento não-farmacológicas para promover qualidade de vida aos pacientes. Ao analisar as evidências dos estudos na área, procura-se explorar os seus benefícios por ser uma prática não-invasiva, de fácil uso e acessível. Portanto, a AA apresenta possibilidade de maior inserção na prática clínica de enfermeiros, visto que pode ser implementada como uma intervenção para o cuidado de idosos com dor.

\section{Conclusão}

De acordo com os estudos selecionados, foi possível evidenciar efeitos positivos na utilização da AA em diversos pacientes que sofrem de dor, tais como idosos oncológicos, indivíduos submetidos a procedimentos cirúrgicos, com dor nas costas, dor lombar e dor no joelho. A AA como PICS, tem-se mostrado eficaz e promissora para o tratamento de diferentes tipos de dor, uma vez que reduziu significativamente os níveis de intensidade da dor, em pacientes que receberam a intervenção comparados ao grupo-controle.

Portanto, sugere-se novos estudos clínicos direcionados aos idosos, a fim de favorecer uma maior profundidade voltada para as particularidades e cuidados específicos desse público. É relevante que os profissionais da saúde desenvolvam novas pesquisas sobre o tema para que ocorram atualizações em pesquisas baseadas em evidências. Ressalta-sea possibilidade de uma maior inserção do enfermeiro nos cuidados relacionados a idosos com dor por meio da AA associada ou não a outros tratamentos.Também espera-se que os resultados deste estudo incentivem a utilização da AA por enfermeiros em sua prática assistencial.

\section{Referências}

Alimi, D. et al. (2003). Analgesic Effect of Auricular Acupuncture for Cancer Pain: A Randomized, Blinded, Controlled Trial. Journal of Clinical Oncology, 21(22), $4120-4126 .<10.1200 / \mathrm{JCO} .2003 .09 .011>$.

Araújo, W. C. O. (2020). Recuperação da informação em saúde: construção, modelos e estratégias. Conv. Ciênc. Inform., 3(2), 100-134.

Aromataris, E. \& Munn, Z. (Editors) (2021). JBI Manual for Evidence Synthesis. https://doi.org/10.46658/JBIMES-20-01.

Artioli, D. P., Tavares, A. L. F. \& Bertolini, G. R. F. (2019). Auriculotherapy: neurophysiology, points to choose, indications and results on musculoskeletal pain conditions: a systematic review of reviews. BrJP, 2(4), 356-361.

Azevedo, A. C. B. et al. (2015). Benefícios das Práticas Alternativas Integrativas e Complementares na Qualidade de Vida da Pessoa Idosa. Acta Ciênc. Saúde, $1(4), 43-59$.

Azevedo, C. et al. (2021). Auriculoterapia em adultos e idosos com sintomas do trato urinário inferior: revisão integrativa. Rev. Esc. Enferm. USP, 55(e03707). https://doi.org/10.1590/S1980-220X2020000503707.

Ministério da Saúde (2020). Práticas Integrativas e Complementares (PICS). https://www.gov.br/saude/pt-br/assuntos/saude-de-a-a-z-1/p/praticas-integrativase-complementares-pics.

Castro, R. M. M. et al. (2020). Utilização da aromaterapia e auriculoterapia como métodos não farmacológicos para alívio da dor em idosos. Braz. J. ofDevelop., 6(8), 60770-60787. <10.34117/bjdv6n8-479>

Contim, C. L. V., Santo, F. H. E. \& Moretto, I. G. (2019). Aplicabilidade da auriculoterapia em pacientes oncológicos: revisão integrativa da literatura. Rev. Esc. Enferm. USP, 54 (e03609), 1-12.

Estores, I. et al. (2017). Auricular acupuncture for spinal cord injury related neuropathic pain: a pilot controlled clinical trial. The J. of Spinal Cord Medicine, 40(4), 432-438. 
Fineout-Overholt E., \& Stillwell S. B. (2011). Asking compelling, clinical questions. In: Melnyk B. M.; Fineout-Overholt, E. Evidence-based practice in nursing \& healthcare. A guide to best practice. Philadelphia: Wolters Kluwer, Lippincot Williams \& Wilkins; 25-39.

Freitas, E. V. \& Py, L. (2017). Tratado de Geriatria e Gerontologia. (4a ed.), Guanabara Koogan.

Instituto Brasileiro de Geografia e Estatística, Agência do IBGE. Projeção da População 2018: número de habitantes do país deve parar de crescer em 2047. https://agenciadenoticias.ibge.gov.br/agencia-sala-de-imprensa/2013-agencia-de-noticias/releases/21837-projecao-da-populacao-2018-numero-de-habitantesdo-pais-deve-parar-de-crescer-em-2047.

Krusche-Mandl, I. et al. (2019). Implementation of Electrical Auricular Acupuncture and Low Frequency Modulated Electric Current Therapy in Pain Management of Patients with Knee Osteoarthritis: A Randomized Pilot Trial. J. Clin. Med.,8(8), 1229. < 10.3390/jcm8081229>

Mendes, K. D. S., Silveira, R. C. C. P. \& Galvão, C. M. (2008). Revisão integrativa: método de pesquisa para a incorporação de evidências na saúde e na enfermagem. Texto Context - Enferm, 17(4), 758-764.

Mao, J. J. et al. (2021). Effectiveness of Electroacupuncture or Auricular Acupuncture vs Usual Care for Chronic Musculoskeletal Pain Among Cancer Survivors The PEACE Randomized Clinical Trial. JAMA Oncol.,7(5), 720-727. <10.1001/jamaoncol.2021.0310>

Moura, C. C. et al. (2018). Ação da auriculoacupuntura em pessoas com dor crônica na coluna vertebral: ensaio clínico randomizado. Rev. Latino-Am. Enferm., 26(e3050). <10.1590/1518-8345.2678.3050>

Moura, C. C. et al. (2019). Acupuntura auricular para dor crônica nas costas em adultos: revisão sistemática e metanálise. Rev. Esc. Enferm. USP, 53. https://doi.org/10.1590/S1980-220X2018021703461

Moura, C. C. et al. (2018). Contribution of Chinese and French ear acupuncture for the management of chronic back pain: A randomised controlled trial Journal of Clinical Nursing, 28, 3796 - 3806. <10.1111/jocn.>

Moura, C. C. et al. (2019). Efeitos da auriculoacupuntura na dor crônica em pessoas com distúrbios musculoesqueléticos nas costas: ensaio clínico randomizado*. Rev. Esc. Enferm. USP,53.<10.1590/S1980-220X2018009003418>

Moura, C. C. et al. (2017). Impactos da dorcrônica na vidadas pessoas e aassistência deenfermagem noprocesso. AvEnferm, $35(1)$, 53 - 62. $<10.15446 /$ av.enferm.v35n1.61006>

Murakami, M., Fox, L. \&Dijkers, M. P. (2017). Ear Acupuncture for Immediate Pain Relief- A Systematic Review and Meta-Analysis of Randomized Controlled Trials. Pain Medicine, 18, 551-564.

Nunes, M. F. et al. (2017). A acupuntura vai além da agulha: trajetórias de formação e atuação de acupunturistas. Saúde Soc., 26(1), 311.

Oliveira, M. M. et al. (2011). Controlando a dor: benefícios da acupuntura auricular e auriculoterapia em idosos. Rev. Tema, Campina Grande, 11(16).

Page, M. J. et al. (2021). The PRISMA 2020 statement: An updated guideline for reporting systematic reviews. BMJ., 372(71), 1-9.

Sator-Katzenschlager, S. M. et al. (2004). The Short- and Long-Term Benefit in Chronic Low Back Pain Through Adjuvant Electrical Versus Manual Auricular Acupuncture.AnesthAnalg, 98,1359 - 1364. < 10.1213/01.ane.0000107941.16173.f7>

Sociedade Brasileira De Geriatria E Gerontologia (2018). Dor: o quinto sinal vital abordagem prática no idoso.

Suen, L. K. P. et al. (2007). Auriculotherapy on low back pain in the elderly. Complem. Therapies in Clin. Pract., 13, 63-69. <10.1016/j.ctcp.2006.10.005>

Ruela, L. O. et al. (2018). Efetividade da acupuntura auricular no tratamento da dor oncológica: ensaio clínico randomizado. RevEscEnferm USP,52(e03402) $<10.1590 /$ S1980-220X2017040503402>

Usichenko, T. I. et al. (2007). Auricular acupuncture for pain relief after ambulatory knee surgery: a randomized Trial. CMAJ,176(2), 179-183. < 10.1503/CMAJ.060875>

Usichenko, T. I. et al. (2005). Auricular acupuncture for pain relief after total hip arthroplasty - a randomized controlled study. Pain, 114(3), 320 - 327. $<10.1016 /$ j.pain.2004.08.021>

Wang, G., Ayati, M. H. \& Zhang, W. (2010). Meridian Studies in China: A Systematic Review. J Acupunct Meridian Stud, 3(1), 1-9.

Whittemore, R. et al. (2014). Methods for knowledge synthesis: An overview.Hear Lung J Acute Crit Care, 43(5), $453-461$.

Zeliadt, S. B. et al. (2020). Patient Feedback on the Effectiveness of Auricular Acupuncture on Pain in Routine Clinical Care: The Experience of 11,406 Veterans. Medical Care, 58(9), suppl. 2. <10.1097/MLR.0000000000001368> 